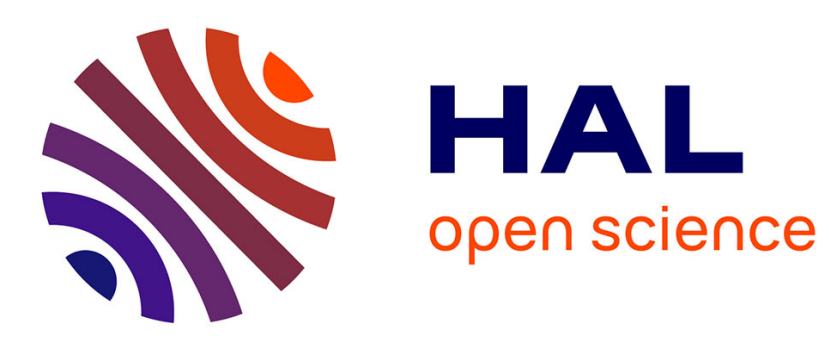

\title{
Segmented Solenoid Coil Antenna for UHF RFID Near-Field Reader Applications
}

\author{
Mossaab Daiki, Etienne Perret
}

\section{To cite this version:}

Mossaab Daiki, Etienne Perret. Segmented Solenoid Coil Antenna for UHF RFID Near-Field Reader Applications. IEEE Journal of Radio Frequency Identification, 2018, 2 (4), pp.210-218. 10.1109/JRFID.2018.2887184 . hal-02054094

\section{HAL Id: hal-02054094 \\ https://hal.science/hal-02054094}

Submitted on 1 Jul 2020

HAL is a multi-disciplinary open access archive for the deposit and dissemination of scientific research documents, whether they are published or not. The documents may come from teaching and research institutions in France or abroad, or from public or private research centers.
L'archive ouverte pluridisciplinaire HAL, est destinée au dépôt et à la diffusion de documents scientifiques de niveau recherche, publiés ou non, émanant des établissements d'enseignement et de recherche français ou étrangers, des laboratoires publics ou privés. 


\title{
Segmented Solenoid Coil Antenna for UHF RFID Near-Field Reader applications
}

\author{
D. Mossaab and E. Perret, Senior Member, IEEE
}

\begin{abstract}
A segmented solenoid coil antenna for UHF nearfield RFID applications is reported herewith for the first time. A large read zone, in comparison to the antenna size is achieved using an antenna made of 4-turns of wire, wrapped around a cylinder of diameter $5 \mathrm{~cm}$ and height $2.6 \mathrm{~cm}$. Similar to HF RFID reader antennas, it has been proved that by increasing the number of turns, it is possible to concentrate and make magnetic field in the antenna surrounding zone uniform, and thus increase the read range. This improvement first modeled in simulations, is then realized and validated experimentally. In the optimum configuration, the maximum read range is increased by $6 \mathrm{~cm}$ in comparison to a single turn planar loop. In addition to enhancement in read performance, this antenna is fabricated on a flexible substrate using a low cost printing process.
\end{abstract}

Index Terms - Reader antenna, RFID UHF, segmentation, near-field, solenoid.

\section{INTRODUCTION}

$\mathrm{R}$ adio Frequency Identification (RFID) is a wireless communication technology that emerged several decades ago. RFID has many advantages that make it useful in many application domains. Advantages of UHF RFID differentiate this technology clearly from other contactless identifies like barcodes [1]-[3]. A basic RFID system essentially consists of a reader and an active or passive transponder/identifier called RFID tag. We focus on passive UHF RFID systems operating in the $868 \mathrm{MHz}$ band, which use fully-passive tags (without any power source). These systems could achieve a read range around $10 \mathrm{~m}$ for typical tags size of $1 \times 9 \mathrm{~cm}^{2}$ [1]. However, some applications like item level tagging, security and access control applications, and products inventory and control of supply chain etc., require identification systems with limited read ranges. This is to accommodate several reading systems and ensure signal security to the data exchanged [1]-[7].

To exploit the best of UHF RFID, for specific applications, new systems based on near-field (NF) communications are envisaged [5]. In such configurations, a limited zone similar to a cube with side length of few dozen centimeters is desired generally.

By using UHF RFID in NF communication scheme, we

M. DAIKI was with Univ. Grenoble Alpes, LCIS, F-26900, Valence, France.

E. PERRET is with Univ. Grenoble Alpes, LCIS, F-26900, Valence, France (e-mail: etienne.perret@lcis.grenoble-inp.fr) and also with Institut Universitaire de France, 75005, Paris, France. could benefit from features like high read rate, cheaper and smaller sized tags. Reader antennas of these systems operate in Rayleigh zone. In which case, data exchange between reader and tags occurs through magnetic or inductive coupling [5] just as in HF RFID. Hence, read zone is directly linked to the magnetic field distribution.

In most cases, read area required (about $15 \times 15 \mathrm{~cm}^{2}$ ) corresponds to the reader antenna surface itself. This results in reader antenna size larger than operating wavelength $\left(\lambda_{0}\right)$. Hence a classical loop with many turns such as in HF RFID can no longer be used [6]-[12] and a specific design approach is required. In fact, considering the ratio of dimension to wavelength, a classic loop antenna will have a non-uniform current distribution that generates a weak and non-uniform magnetic field surrounding the loop [6]-[12]. Indeed, the current flowing in the loop nulls at every half-wavelength and changes direction. To ensure a uniform current distribution along loop circumference, several techniques could be used [6]-[12]. A popularly used technique is segmentation [6]-[12]. In which, the loop is formed by at least two parallel coupled segments to enhance the uniformity of current density along the loop. In general, the segment length is less than $\lambda_{0} / 4$ to ensure quasi constant current density on each segment [6][12].

In magnetostatics, it is well-known that by increasing the number of turns of a loop, the magnetic field magnitude can be enhanced. This principle is used in HF RFID to design tags as well as reader antennas [2]. A coil in solenoid form allows concentrating the magnetic field in a defined direction in space (along its axis). This is mostly preferred for fabrication of inductors and transformers [2], [13]-[17] at low frequency. Some accurate models are also available in HF band [13][15]. At higher frequency, an inductor is made of a stack of 12 layers and is characterized at $5 \mathrm{GHz}$ [16]. The fabrication is complex, costly and incompatible with the realization of a NF UHF RFID antenna.

In this article we present for the first time, a technique to transpose the solenoid concept to design a NF UHF RFID reader antenna with enhanced read zone (see Fig. 1). Indeed, by increasing the number of turns in comparison to a classic loop antenna already used, it is possible to extend the read zone without increasing the antenna surface. The volume outside and also inside the solenoid antenna (Fig. 1) could be used to read NF UHF RFID tags. 
$=$ reading zone

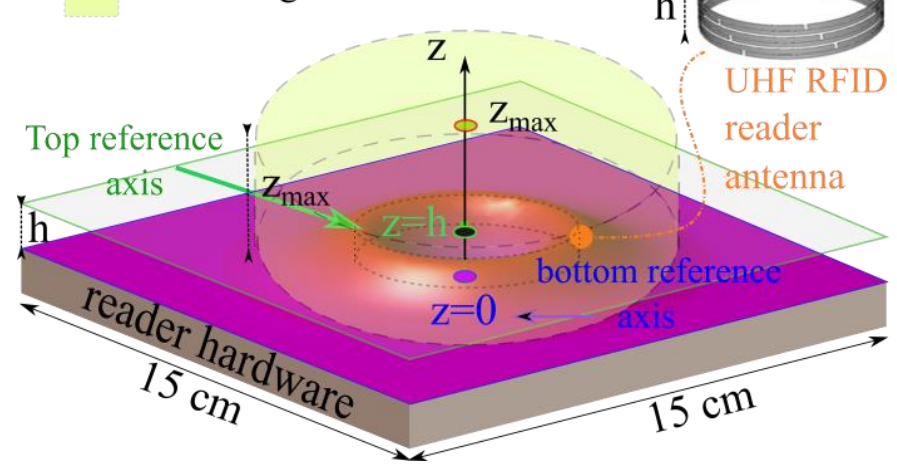

Fig. 1. The proposed solenoid reader antenna and it corresponding read zone which consists of the volume inside the solenoid and also a volume surrounding the antenna.

Due to ratio between wavelength and length of the strip used for realization of the solenoid antenna, the segmentation technique is used to ensure a homogenous current distribution along the solenoid antenna. With such antenna dimensions, the use of a solid strip, like it is often done in HF, would not produce a homogeneous field distribution. We will see that the segmentation is a flexible technique that could be used to design antennas for NF applications with dimensions comparable to the wavelength and by quipping the same antenna shapes (here a solenoid) than the ones generally used at lower frequency within the quasistatic approximation. We will present through an example that the magnetic field generated by a segmented solenoid antenna is proportional to it number of turns, like in a lower frequency (HF band). In addition, a low cost realization technique is also discussed and implemented. The proposed solenoid antenna is realized on a PET substrate by using a classic printing technique similar to the one used for fabrication of RFID tag antennas. Section II presents the topology of proposed solenoid antenna and impact of number of turns on the magnetic field. In section III, we present some relevant simulation results of a 4-turn segmented solenoid antenna intended for NF UHF RFID applications. Then, in section IV, we describe realization process and measurement/experimental procedure. Finally, a comparison of experimental results with a commercial NF UHF RFID reader antenna is presented.

\section{EFFECT OF SOLENOID TURNS ON MAGNETIC FIELD}

A segmented circular solenoid with different numbers of turns is studied as shown in Fig. 2. The 1-turn segmented

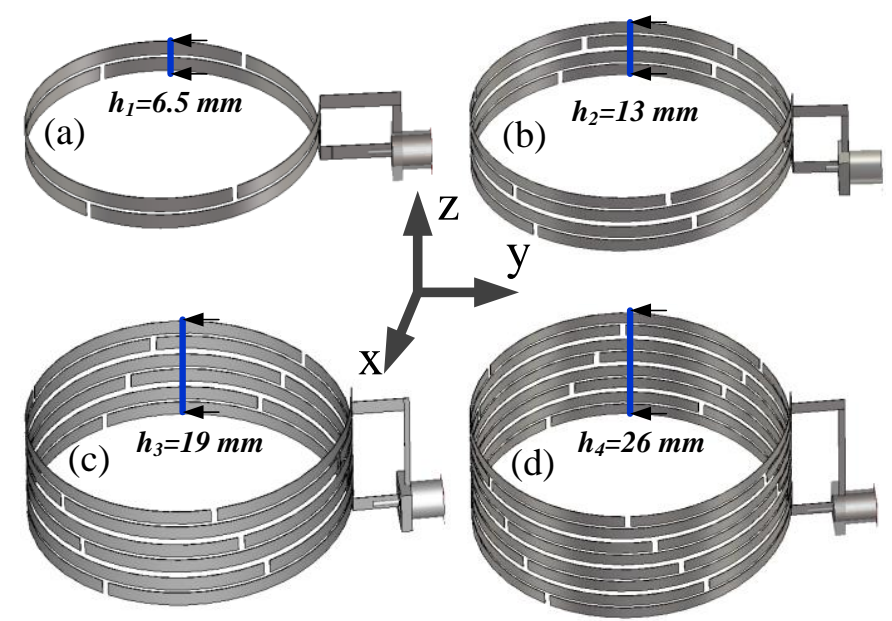

Fig. 2. Segmented solenoid with different numbers of turns: (a) 1-turn, (b) 2 turns, (c) 3-turns, (d) 4-turns.

TABLE I. SEGMENTED SOLENOID DIMENSIONS

\begin{tabular}{ccc}
\hline \hline Solenoid & Solenoid height $(\boldsymbol{h})$ in $\mathrm{mm}$ & Total length in $\mathrm{mm}$ \\
\hline 4 turns & 26 & $628=1.82 \lambda_{0}$ \\
3 turns & 19 & $471=1.3 \lambda_{0}$ \\
2 turns & 13 & $314=0.9 \lambda_{0}$ \\
1 turn & 6.5 & $157=0.45 \lambda_{0}$ \\
\hline \hline
\end{tabular}

solenoid is similar to a classic loop antenna. However, the structure (see Fig. 2a) is not planar as the one described in [7].

We fix loop perimeter as $0.45 \lambda_{0}$ at $868 \mathrm{MHz}$ in the 1-turn solenoid, for a diameter $(D)$ of $5 \mathrm{~cm}$, to ensure uniform current density on the antenna, without change in directions. In such configuration, the segmentation technique does not show any significant positive impact on the magnetic field distribution around the loop. However, when the numbers of turns are increased, the segmentation technique must be used to maintain a uniform current density along the loop. Table I summarizes the total lengths of four solenoids presented in Fig. 2. When we unroll the wires of different solenoids, the total length varies between $0.45 \lambda_{0}$ and $1.82 \lambda_{0}$.

Fig. 3a presents a 4-turn segmented solenoid antenna and Fig. $3 \mathrm{~b}$ a non-segmented one, both with a total length of $1.82 \lambda_{0}$.

The magnitude of magnetic field generated by these two antennas is plotted Fig. 4. It is clear from this that the magnetic field surrounding 4-turn segmented solenoid antenna is much stronger and uniform in comparison to unsegmented one. 
Segmentation parameters used for the solenoid are given in Fig. 3a. To make segmented structure, the metallic strip that constitutes the loop needs to have at least two turns of segments in a staggered arrangement. The objective of this specific arrangement is to obtain a maximally uniform current density along the strip. Each segment can be modeled by a RLC circuit as discussed in [12]. The inductance $L_{m o d}$ corresponds to presence of the small line which completes the loop. With segmentation technique, gap between each small line introduces a capacitance $C_{m o d}$ into each segment. The electrical resistance $R_{\text {mod }}$ represents losses in the structure. Thus depending on $L_{\text {mod }}$ and $C_{\text {mod }}$ the equivalent series RLC circuit (see Fig. 3c) can resonate at the desired operating frequency (UHF band in this case). At this specific frequency the phase shift through this segment is zero Hence a uniform current could be observed over the antenna [12], due to absence of phase delay between two adjacent segments. Note that to obtain such specialty, the dimensions of the segments play a significant role. To maintain such a current along the line, the segment length needs to be less than $\lambda_{0} / 4$. The space gap between two segments or between the two turns need to be lower than $0.5 \mathrm{~mm}$ to ensure good coupling [6]-[12]. By simply following these instructions, it is possible realize large structures relative to wavelength, for a desired topology. We show for the first time that a 3D structure could be obtained with such an approach.

In Fig. 4a, we could see that current flows in one direction along the segmented solenoid. Conversely, nulls and direction changes of the current along the solid solenoid (each half wavelength) is observed in Fig. 4b. This means that, for segmented case, the magnitude of magnetic field is more uniform and stronger than for un-segmented solenoid (Figs. $4 \mathrm{c}$, and $4 \mathrm{~d})$. This comparison highlights the benefit of segmentation technique in maintaining a uniform current density for large antennas.

Segmented circular solenoids with various numbers of turns are then designed to evaluate the effect of number of turns on magnetic field magnitude (see Fig. 2). Segmentation settings given in Fig. 3a are kept the same for all solenoids. Fig. 3c shows measured S11 parameter of the segmented circular solenoids presented in Fig. 3a. Despite of negligible imperfections in realization of the 3D antenna with a flexible substrate, a good matching is obtained in the European UHF band. Segmented solenoids are intrinsically resonant structures, and as mentioned previously. Entire segments that constitute the solenoid could be modeled as a serial RLC circuit. Note that two lines have been added in order to connect the solenoid to the SMA connector. These lines (see $L_{a}$ on Fig. 3a) are longer in the 3D structure compared to a planar one [12] and affects the resonant frequency of entire structure. Indeed as shown in Fig. 3c, an additional inductance $L_{L A}$, corresponding to this extra line $L_{a}$, should be considered. By optimizing the length $L_{a}$ (see Fig. 3a) between the solenoid and (SMA) connector (see Fig. 3c where S11 simulation results for different $L_{a}$ values are plotted), i.e. by optimizing the inductance $L_{L A}$, these solenoid antennas could be easily impedance matched (at $868 \mathrm{MHz}$ in this case). Classically, this method needs significant simulation time, because for each $L_{a}$ value, simulation of the entire structure (solenoid with the connector) should be carried out, and this approach must be repeated for each configuration. To mitigate this issue, an ideal lumped matching circuit has been added, with which the length $L_{a}$ does not vary, in CST simulation model. In this way, a perfect matching can be obtained for the desired frequency. A similar reflection coefficient for the four studied structures is thus obtained, which makes easier to compare the antenna performance. The normal component of magnetic field $\left|H_{z}\right|$ along the solenoid axis $(z)$ of these four solenoids is presented in Fig. 5. As expected, the increase in number of turns enhances the magnetic field magnitude surrounding the solenoid. This is typically the case when we consider the field on top of each solenoid, which could correspond to the beginning of the read area for some applications.

In the table presented in Fig. 5, magnetic field amplitude $\left|H_{z}\right|$ has been evaluated at $4 \mathrm{~mm}$ above the solenoid top surface in the four cases. Again the magnetic field amplitude is seen to increase with number of turns. As shown Fig. 1, we could see that by increasing the number of turns and in turn the solenoid height, a read area volume corresponding to the area inside and also outside the solenoid is increased.

In order to obtain the relationship between the number of turns and the magnetic field $\left|H_{z}\right|$, an analytical model introduced in [18] is used. This model could be used to evaluate the magnetic field of a circular loop with constant
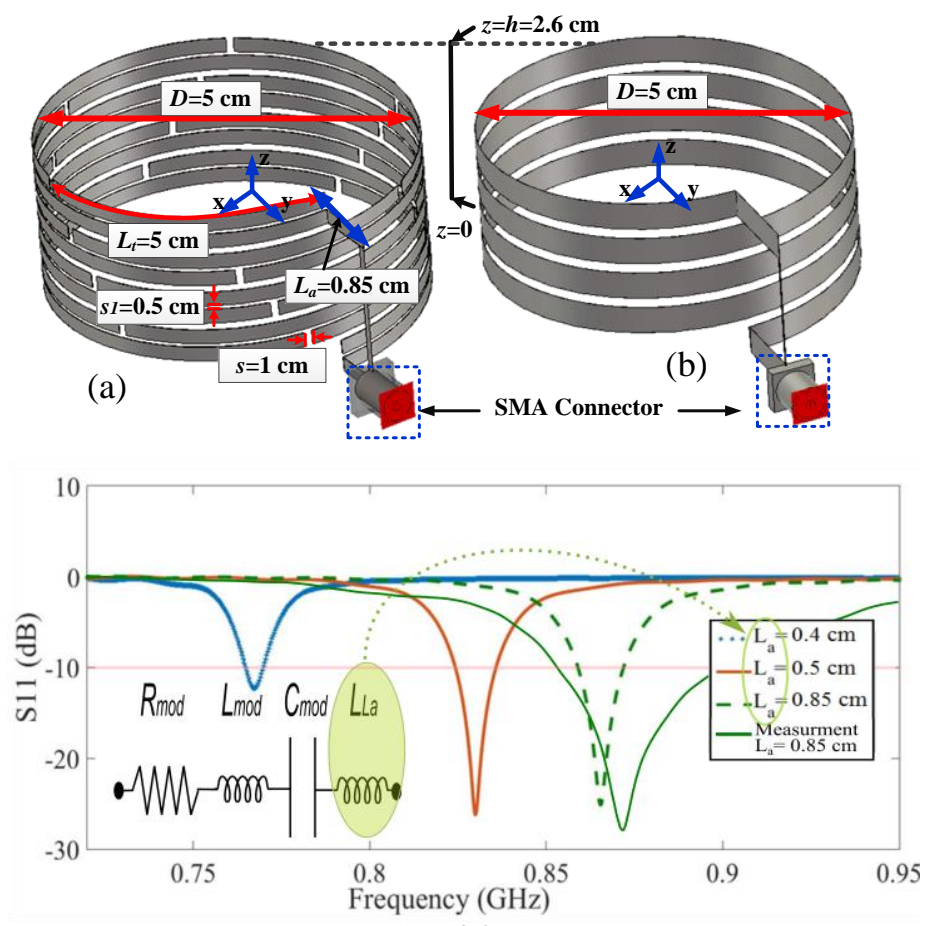

(c)

Fig. 3. Solenoid designs: (a) 4-turn segmented solenoid, (b) 4-turn solid solenoid. (c) Measured S11 parameter (in the anechoic chamber) and simulated impact of length $L_{a}$ on antenna resonance for the 4-turn segmented solenoid shown in Fig. 3a. Inset shows the segmented circular solenoid's equivalent circuit.

current and arbitrary dimensions in comparison to wavelength. The formula is valid for both far-field and NF regions. In this 


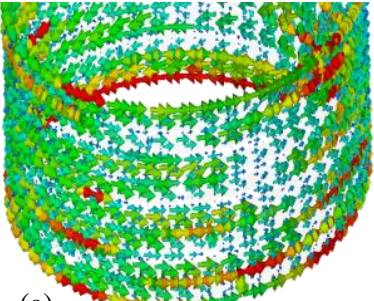

(a)

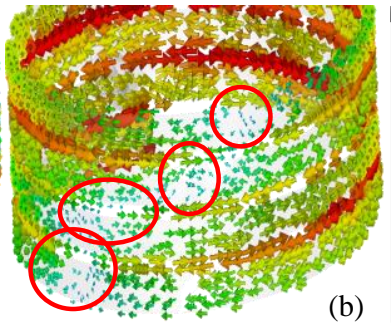

(b)

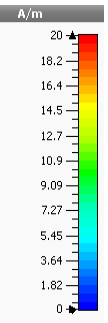

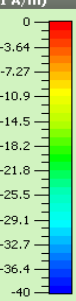

(d)

Fig. 4. Impact of segmentation technique on current density and magnetic field distribution at $868 \mathrm{MHz}$ : (a) 4-turn segmented solenoid, (b) 4-turn solid solenoid (red circles show current null zones). Normal component of the magnetic field $\left|H_{z}\right|$ at $z=5 \mathrm{~cm}$ for: (c) segmented solenoid, and (d) solid line solenoid. An input power of $1 \mathrm{~W}$ is used in simulation.

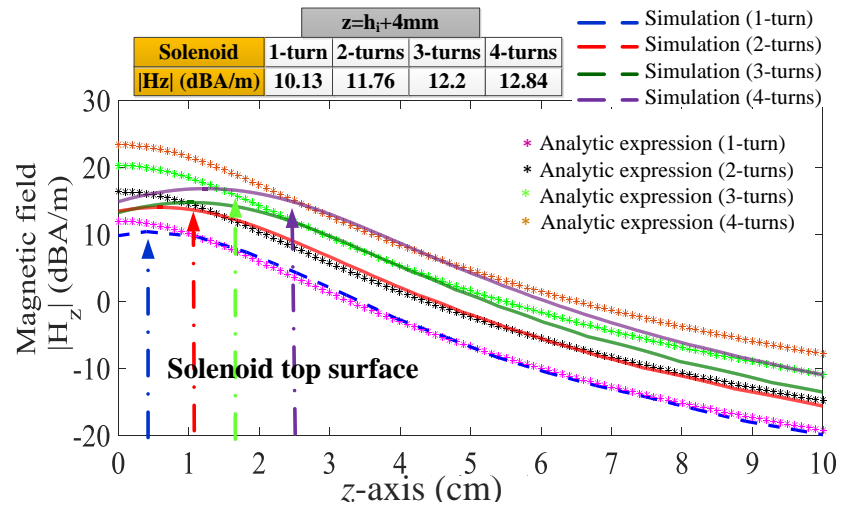

Fig. 5. Normal magnetic field component $|H z|$ computed along the solenoid axis. Comparison between the analytic model (dotted line), and the simulation results (solid line) for solenoids with different number of turns. Simulation input power $=1 \mathrm{~W}$.

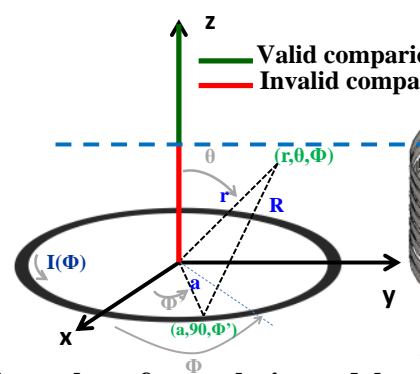

Planar loop for analytic model

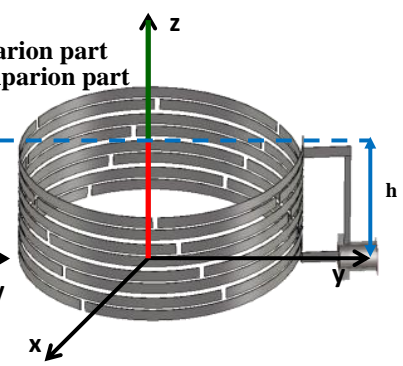

4-turn segmented solenoid

Fig. 6. Proposed method for solenoid current extraction.

the radial component of magnetic field is given as (1)

$$
\begin{aligned}
& H_{r} \\
& =\frac{\beta(\beta a)^{2}}{2 j} I \cos \theta \cdot \exp (-j \beta R) \sum_{m=1}^{\infty} \sum_{k=0}^{2 m-2} D_{m k}^{2 \frac{\left(\beta^{2} a \cdot r \cdot \sin \theta / 2\right)^{2 m-2}}{(\beta R)^{2 m+k}}} \\
& \beta=\frac{2 \pi}{\lambda}, R=\sqrt{r^{2}+\mathrm{a}^{2}-2 \operatorname{ar} \sin \theta \cos \Phi}
\end{aligned}
$$

$$
\begin{array}{r}
D_{m, k}{ }^{1}=\frac{(2 m+k-1) !(-1)^{m}}{(2 j)^{k}(2 m-k-1) ! k ![(m-1) !]^{2} m} \\
D_{m, k}{ }^{2}=m D_{m, k}{ }^{1} \text { for } m \geq 1, k>0 \\
D_{m, 0}{ }^{1}=\left\{\begin{array}{cc}
-1 & \text { for } m=1 \\
-\frac{1}{m(m-1)} D_{m-1,10}{ }^{1} & \text { for } m>1
\end{array}\right.
\end{array}
$$

Where $I$ is the current along the loop, $a$ is the radius, and $(\theta, r, \Phi)$ are the spherical coordinates of the point where field is to be computed, as described in Fig. 6. In this model, current is considered constant along the loop. So, (1) could be applied to the segmented solenoid.

It is clear from this expression that magnetic field magnitude is proportional to current $I$. By choosing the current value, it is possible to fit the simulation curves obtained from CST simulations with (1) for the four different configurations. Here we focus on the radial component $H_{r}$ computed at $z=r$, $\theta=0$ to obtain $H_{l}=H_{z}$. Comparison among the curves simulated in CST and the ones computed using (1) is shown in Fig. 5. As we can see, both the curve families are perfectly superposed in the zone outside the solenoids $\left(z>h_{i}\right.$, where $i$ indicate the number of turns). This model of the loop does not take its thickness into account. So this comparison is not valid for $z$ positions inside the solenoids (Fig. 6).

Extracted values of current $I_{i}$ (where $i$ indicate the number of turns) are given in Fig. 7. The relationship between current value $I_{I}$ extracted for single turn solenoid and current values corresponding to multiple turns $\left(I_{i}, i=\{2,3,4\}\right)$ are also given in Fig. 7. From the expressions describing proportional variations between the current value and the magnetic field, we could see that up to the 4-turn configuration, results are close to values corresponding to a linear variation.

So, it is possible to predict the field outside the solenoid using (1). In this the current value is estimated as, number of turns times current of a single loop $I_{l}$. This result is quite similar to the magnetostatic range. In such a case, magnetostatic field magnitude along the solenoid axis is proportional to number of turns.

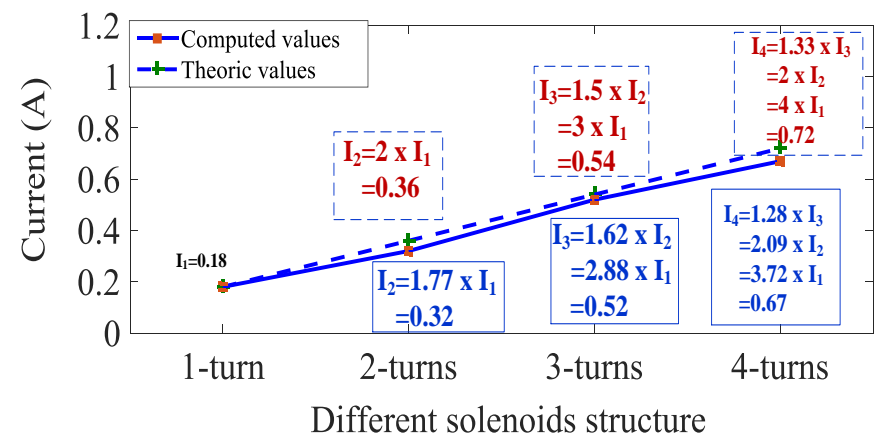

Fig. 7. Computed current values in different segmented solenoids, where $I_{i}$ is the current flowing in the $i^{\text {th }}$-turn(s) of a segmented solenoid. Simulations (solid curve) and Theoretical calculation (1) (dash curve). 


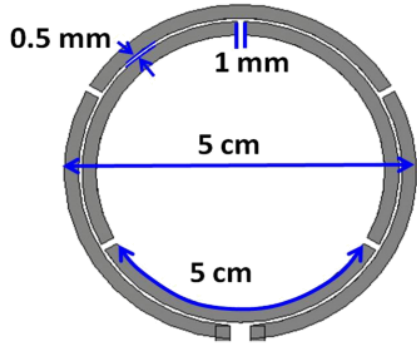

Fig. 8. Planar segmented loop antenna realized on a $0.8 \mathrm{~mm}$ thick FR-4 substrate.

Finally, the average current value for the 1-turn segmented solenoid is extracted directly from CST. A value of $0.26 \mathrm{~A}$ is obtained and this is close to $0.18 \mathrm{~A}$ which is already extracted by fitting the magnetic field curve of 1-turn segmented solenoid to the analytic model [18]. The slight difference between two values could be justified with the fact that (1) is obtained from a linear current density model and it does not take into account the section of conducting wire. In summary, we show that by increasing the number of turns, the magnetic field surrounding the solenoid can be increased as well. The 4turn configuration is the best compromise between magnetic field enhancement and realization complexity. One should also note that with the segmentation technique, homogenous field distribution like the one obtained with a solenoid shape can be easily achieved when dimensions of the structure are in same order of magnitude as the wavelength. The relation between field distribution and the antenna shape is similar to what is classily known about conventional HF coupling coils, which is generally called the quasistatic approximation.

\section{NF UHF RFID READER ANTENNA}

The 4-turn circular segmented solenoid antenna introduced in section II is used for NF RFID UHF applications. The entire structure is simulated using CST microwave studio, including the SMA connector to account for the effect of asymmetric excitation produced. In contrary to the previous section, the antenna is matched by optimizing the length $L_{a}$ (Fig. 3a), without using an ideal matching circuit. The operating frequency chosen is $868 \mathrm{MHz}$ (European UHF RFID band). The simulated reflection coefficient is around $-25 \mathrm{~dB}$ at $868 \mathrm{MHz}$ with $10 \mathrm{~dB}$ return loss bandwidth of $13 \mathrm{MHz}$ (859$872 \mathrm{MHz}$ ). The simulated current density is presented in Fig. 4a. As mentioned previously, the current is quasi uniform and maintains same direction along the antenna.

The segmented solenoid antenna is compared to a segmented planar loop antenna of diameter $5 \mathrm{~cm}$ (Fig. 8), which is modeled with the same segmentation parameters explained previously (Fig. 3a). The loop circumference is less than half wavelength for a diameter of $5 \mathrm{~cm}$, thus a solid loop could also maintain a uniform current. However, we apply the segmentation technique with the same parameters as in the solenoid case to draw a comparison between the two segmented structures. In this simulation, the excitation signal amplitude is fixed at $23 \mathrm{dBm}(\approx 200 \mathrm{~mW})$. This value corresponds to the reader power level that will be used with the experimental measurement part. Curves in Fig. 9 show the variation of normal component of magnetic field $\left(H_{z}\right)$ of the solenoid and the planar loop along $z$-axis at the center of structure $(x=0, y=0)$ and also along the $x$ and $y$ axis. Please note that, the magnetic field $\left(H_{z}\right)$ along $x$ and $y$ axes are different due to the presence of connector and feed cable along the $\mathrm{Y}$ direction. When we look at the evolution of field as a function of $z$, for both configurations, we could see that the maximum is reached in the antenna symmetry planes. $13 \mathrm{dBA} / \mathrm{m}$ at $z=0$ for the planar loop and $3 \mathrm{dBA} / \mathrm{m}$ at $z=h / 2$ for the solenoid.
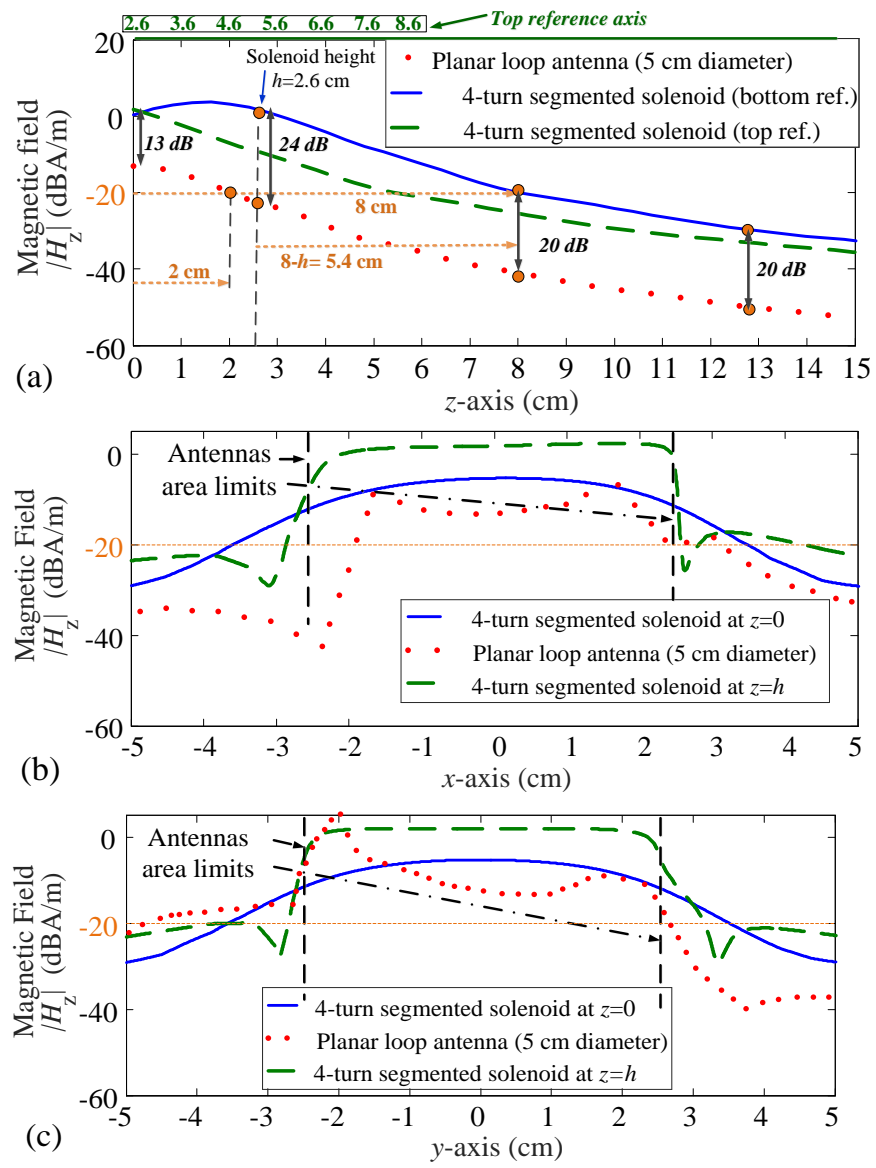

Fig. 9. Comparison of magnetic field component $\left|H_{z}\right|$ of the planar segmented loop $(D=5 \mathrm{~cm})$ and the 4-turn segmented solenoid antenna: (a) along $z$-axis at $x=y=0$ (the "top reference axis" is the coordination system $x=0, y=0, z=h$, i.e. when the relative $z$ origin " 0 " is moved to $z=h$, see Fig. 1), (b) along $x$-axis at $z=y=0$, and (c) along $y$-axis at $x=z=0$.

The extension in $z$ direction of the solenoid ensures better volume distribution of the field, allowing a more homogeneous distribution in a larger area. Indeed, at $h=2.6 \mathrm{~cm}$ the magnetic field of solenoid is $24 \mathrm{~dB}$ above what is created by the plane loop. The difference is reduced to $20 \mathrm{~dB}$ for a height $z$ greater than or equal to $8 \mathrm{~cm}$. It is clear from results presented Fig. 9 that the central axis of solenoid is the best zone to detect NF RFID tags.

The extension in $z$ direction of the solenoid ensures better volume distribution of the field, allowing a more homogeneous distribution in a larger area. Indeed, at $h=2.6 \mathrm{~cm}$ 
the magnetic field of solenoid is $24 \mathrm{~dB}$ above what is created by the plane loop. The difference is reduced to $20 \mathrm{~dB}$ for a height $z$ greater than or equal to $8 \mathrm{~cm}$. It is clear from results presented Fig. 9 that the central axis of solenoid is the best zone to detect NF RFID tags.

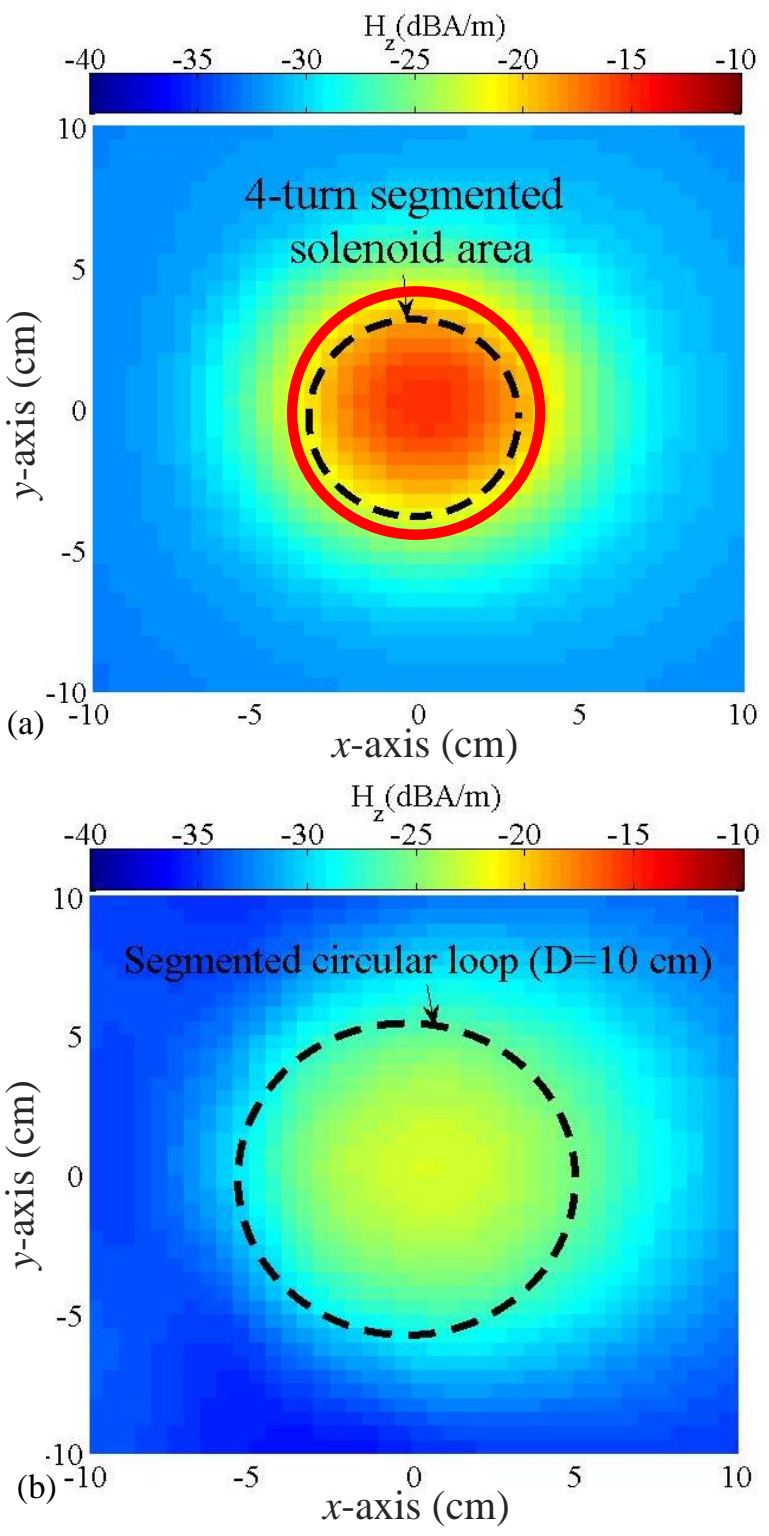

Fig. 10. Magnetic field distribution $|H z|$ at $\mathrm{z}=7 \mathrm{~cm}$ at $868 \mathrm{MHz}$ : (a) segmented solenoid antenna, (b) segmented planar circular loop antenna $(D=10 \mathrm{~cm})$ [7]. *red circle in Fig. 10a delimits the zone where $\left|H_{z}\right| \geq-20 \mathrm{dBA} / \mathrm{m}$.

Again in Fig. 9a, considering the solenoid bottom as reference, the maximum read range is $8 \mathrm{~cm}$, which represent an increase of $6 \mathrm{~cm}$ in comparison to a single turn planar loop $(2 \mathrm{~cm})$, if we consider a threshold level of " $-20 \mathrm{dBA} / \mathrm{m}$ " as the minimum magnetic field amplitude to detect a NF RFID tag [7]. In this case, the solenoid provides four times the read range of a planar loop, according to obtained magnetic field amplitude. Now, if the top of solenoid is taken as reference (see the green curve in Fig. 9a, z-axis corresponding to this specific reference is given at the top of figure), the maximum read range is equal to $5.4 \mathrm{~cm}(8 \mathrm{~cm}-2.6 \mathrm{~cm})$. Which is still greater (more than two times) the read range of planar segmented loop shown in Fig. 9a. We also observe that the magnetic field is $13 \mathrm{~dB}$ higher, in case of the solenoid, when the centers of both structures are considered. Now, if we assume that for specific reasons of application, it is not possible to allow reading of tags inside the solenoid, it is interesting to compare the magnetic field amplitude at the top of the solenoid, with the amplitude just above the planar loop in $x-y$ plane. We could see (in Fig. $9 \mathrm{~b}$ and c), that if the "- 20 $\mathrm{dBA} / \mathrm{m}$ " criterion is used, it is still possible to read tags outside the solenoid for a height $z$ (between 0 and $2.6 \mathrm{~cm}$ ).

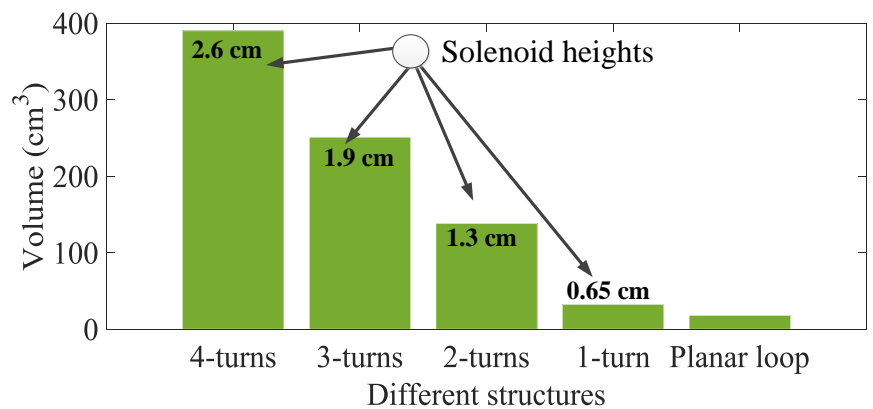

Fig. 11. Simulated read volume for 1, 2, 3 and 4-turn segmented solenoid and planar segmented loop configuration $(\mathrm{D}=5 \mathrm{~cm})$ at input power level of $23 \mathrm{dBm}$.

Fig. 10, shows the magnetic field distribution in $x-y$ plane at $z=7 \mathrm{~cm}$ for the 4-turn segmented solenoid and the segmented planar loop antenna described in [7], with a diameter of $10 \mathrm{~cm}$. In this figure, an area of $20 \times 20 \mathrm{~cm}^{2}$ surrounding the antenna is considered and dotted lines represents the antenna surfaces. . An enhancement of around $5 \mathrm{~dB}$ in magnitude of magnetic field (on a surface greater than the antenna shape) is observed for the solenoid, in comparison to the segmented planar loop. In summary, for NF UHF RFID application, that requires a

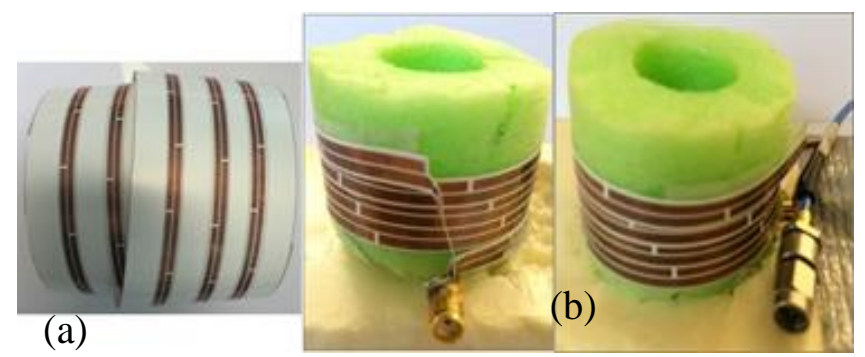

Fig. 12. (a) Segmented copper lines printed on PET substrate, (b) prototype of the segmented solenoid antenna.

maximum range less than $10 \mathrm{~cm}$, the segmented solenoid presents a good tradeoff between read performance and compactness.

In Fig. 11, the simulated read volume of different solenoids $(1,2,3$ and 4-turns) and segmented planar loop $(D=5 \mathrm{~cm})$ is presented to make a comparison among volumes obtained with a power level of $23 \mathrm{dBm}$. This volume is evaluated by computing the read surface at different $z$ planes. Here, the read surface corresponds to an area where the magnetic field magnitude is greater than $-20 \mathrm{dBA} / \mathrm{m}$. In Fig. 11, we could see that read volume of planar segmented loop and 1-turn segmented solenoid is comparable. With this analogy, the read volume increases with the number of turns and a desired optimization could be thus achieved. This is particularly 
applicable when the reading could be allowed in practice inside the solenoid as described in Fig. 1. If we consider that reading could not be implemented in application inside the solenoid, for the 4-turn configuration, a read volume of $331 \mathrm{~cm}^{3}$ is obtained. The segmented solenoid coil is made of four turns of segmented line printed on PET substrate as shown in Fig. 12. This line is realized by low cost ink-jet flex printing technique [19], [20]. The line is wrapped around a hollow cardboard circular cylinder of diameter $5 \mathrm{~cm}$ to obtain the simulated solenoid shape. The SMA connector is directly soldered on to the solenoid (Fig. 12b).

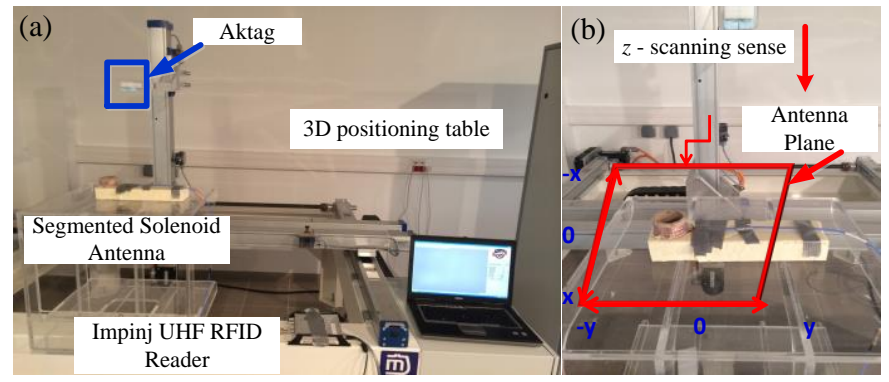

Fig. 13. (a) Measurement setup, (b) scanning procedure.

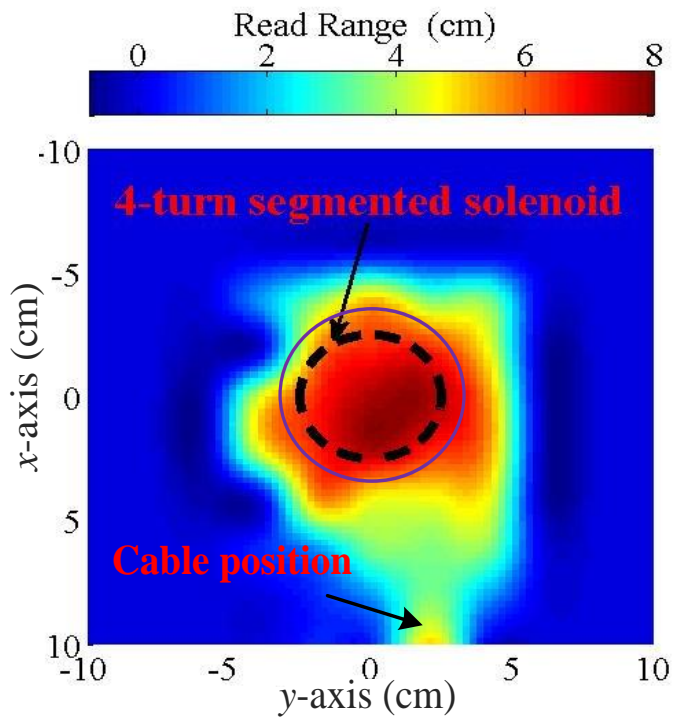

Fig. 14. Cartography result: read zone of the segmented solenoid antenna using an Aktag. *purple circle delimits the zone where the read range is higher than $7 \mathrm{~cm}$. *dotted black circle delimits solenoid antenna surface.

For NF UHF RFID applications, it is interesting to measure the maximum read range and read zone. The measurement setup used is shown in Fig. 13a. It comprises of an Impinj RFID Reader (European band) [21], a 3D positioning table, the segmented solenoid coil antenna, and a NF RFID AKtag tag [22]. This tag antenna is in the form of a loop of $1 \mathrm{~cm}^{2}$. The 3D positioning table is controlled using a MATLAB code, which allows for moving the tag automatically between different positions in space. For each position of the tag, the RFID reader indicates whether the tag is detected or not in order to evaluate the maximum read range (Fig. 13b). To avoid spurious reading, the tag is positioned exactly towards the reader antenna in all cases.
The reader antenna is placed in $x-y$ plane where $z=0$. For each position of this surface, the $z$ distance between tag and reader is varied. Like in simulation studies, the $z=0$ reference corresponds to bottom of the solenoid. The reader power is fixed at $23 \mathrm{dBm}$ for all cases. A surface of $20 \times 20 \mathrm{~cm}^{2}$ centered on the solenoid antenna is scanned and a maximum read range of $8 \mathrm{~cm}$ is observed as shown in Fig. 14. It is interesting to compare the magnetic field simulation (Fig. 10a) with the experimental results in Fig. 14In Fig. 10a, at $z=7 \mathrm{~cm}$, the expected read zone (obtained with the $-20 \mathrm{dBA} / \mathrm{m}$ criterion) is comparable to the read zone of $7 \mathrm{~cm}$ obtained in experiment (red circle in Figs. 10a and 14). The imperfection in realization lead to a slightly off-circular shape of the solenoid and the magnetic field is impacted by this, as expected. Also please note that the 3D displacements are done with reference to a Cartesian coordinate system which necessarily introduces a significant truncation error when the measured field has a circular shape. This could justify in part the difference between measured and simulated fields. However, we obtain a concentric distribution of the field with maxima at the center of the solenoid. Moreover, Fig. 14 also shows the impact of feed cable on magnetic field distribution due to local perturbation, which is also a reason for the difference quoted

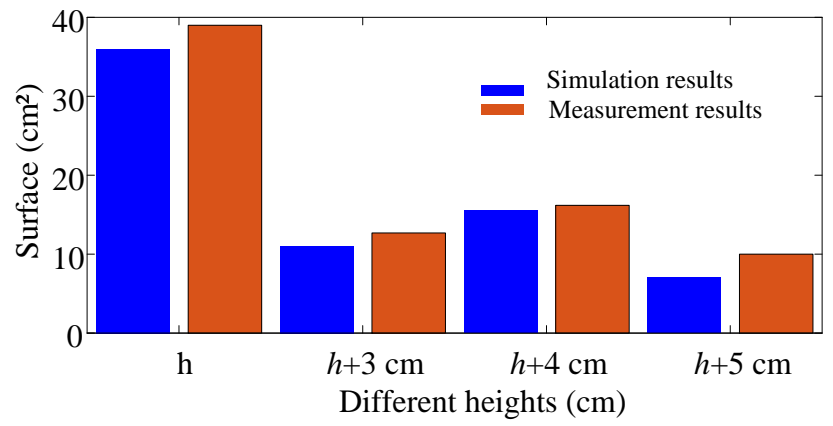

Fig. 15. Comparison between the read surfaces at different heights for the 4-turn segmented solenoid, obtained with experiment and simulation $(h=$ $2.6 \mathrm{~cm}$ ). The simulated read surface is determined with the $-20 \mathrm{dBA} / \mathrm{m}$ criterion on the magnetic field $\left|H_{z}\right|$.

above. Indeed, in Fig. 10a, the simulation results show a perfect circular shape of the magnetic field around the antenna. In Fig. 14 (experimental results), we could also see that outside the solenoid, read range is greater than " $0 \mathrm{~cm}$ " in the near field zone of the cable (around $2 \mathrm{~cm}$ ).

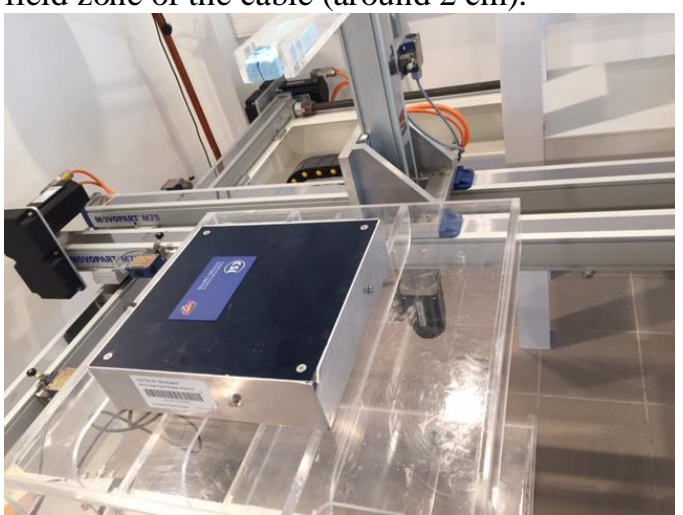

Fig. 16: Characterization of a commercial NF UHF RFID antenna. The antenna is a loop of diameter $10 \mathrm{~cm}$, based on a comparable design of the one simulated Fig. 10b. However the loop is surrounding by a metallic box.

To realize a quantitative comparison, the read surface at different heights is evaluated in simulation (with the - 
$20 \mathrm{dBA} / \mathrm{m}$ criterion) and from experimental results and is presented in Fig. 15.

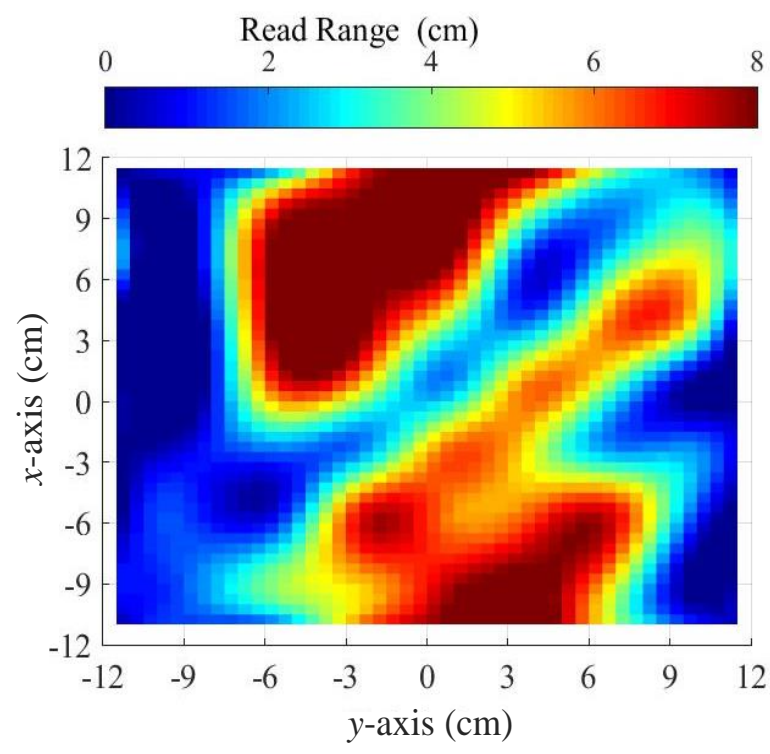

Fig. 17: Cartography result: read zone of the commercial reader (loop) antenna (Fig. 16) using Aktag and an input power of $17 \mathrm{dBm}$.

The simulated and measured read zones shows good agreement. Note that the maximum read range for the $10 \mathrm{~cm}$ diameter loop in [7] characterized with the same input power level $(23 \mathrm{dBm})$ is about $6.5 \mathrm{~cm}$, which is $1.5 \mathrm{~cm}$ less than the solenoid antenna of $5 \mathrm{~cm}$ diameter.

The performance analysis of a commercial NF UHF RFID antenna is carried out as shown in Fig. 16. This is a loop antenna encapsulated into a metallic box with a total size of $23 \times 23 \mathrm{~cm}^{2}$ and a height of $4 \mathrm{~cm}$ [23]. The reader output power is fixed as (for regularization with the solenoid experiment, taking into account the antenna gain which is 6 $\mathrm{dBi}$ - i.e. $6 \mathrm{~dB}$ more than the 4-turn solenoid antenna) 17 $\mathrm{dBm}$, and the read volume using the same tag [22] has been evaluated. The results are given in Fig. 17. We see that the presence of metallic box has a significant impact on magnetic field repartition. The read volume is higher in comparison to the 4-turn segmented solenoid antenna (it is composed of two zones where the read range reaches $8 \mathrm{~cm}$ ), however the maximum read range is comparable $(8 \mathrm{~cm})$. Like in Fig. 11, the read volume is obtained by computing the read surface at different $\mathrm{z}$ planes. A volume of about $1020 \mathrm{~cm}^{3}$ has been obtained for the commercial antenna which is greater (around two fold) than that for the 4-turn solenoid $\left(443 \mathrm{~cm}^{3}\right.$ if reading inside the solenoid is considered and $392 \mathrm{~cm}^{3}$ otherwise). However, the solenoid volume $\left(51 \mathrm{~cm}^{3}\right)$ is very small in comparison to the commercial reader $\left(2116 \mathrm{~cm}^{3}\right)$.

\section{CONCLUSION}

In this paper, a segmented solenoid antenna for NF UHF RFID applications has been introduced for the first time. It has been shown that (up to 4 turns), the current flowing through the solenoid, and hence the value of magnetic field generated around the antenna, is proportional to the number of turns. A 4-turn segmented solenoid antenna has been realized and characterized. This antenna has a low fabrication cost and good read performance comparable to commercial NF UHF antenna which is less compact $\left(2116 \mathrm{~cm}^{3}-\right.$ compared to $51 \mathrm{~cm}^{3}$ the for the solenoid antenna). The 3D read zone obtained in EM simulation is validated by experiments. The proposed antenna is easy to implement in practice since the shape is simple. The read zone can be easily optimized by changing the solenoid dimensions. For instance, larger read ranges can be obtained just by increasing the height of the solenoid. This could be done by increasing the number of turns. Last but not least, the experimental characterizations are conducted in quasi-real environment and agree well with the simulation results. However, the simulation of such segmented solenoid requires large computing resources and is the reason for which, in this paper a small solenoid with a diameter of 5 $\mathrm{cm}$ is studied. Solenoid antennas with higher diameter (such as $10 \mathrm{~cm}$ ) would provide much more performance, and could be more relevant for practical RFID applications. The approach for this would be same as the explained segmentation technique. Here we show through an example, that a solenoid with very same structure and dimensions but optimized using the segmentation technique could be considered as a good candidate for UHF applications. This is an interesting technique for NF applications.

\section{ACKNOWLEDGMENT}

The authors would like to thank RFTLab for providing the 3D positioning table for experimental studies.

\section{REFERENCES}

[1] Z. Min, L. Wenfeng, W. Zhongyun, L. Bin, and R. Xia, “A RFIDbased Material Tracking Information System," in 2007 IEEE Int. Conf. on Automation and Logistics, 2007, pp. 2922-2926.

[2] K. Finkenzeller, RFID Handbook: Radio-frequency identification fundamentals and applications. Wiley, 1999.

[3] P. Adamcova and Z. Tobes, "UHF RFID Technology and its Applications," Int. Conf. Radioelektronika, 2007, pp. 1-5.

[4] A. Michel, P. Nepa, X. Qing, and Z. N. Chen, "Considering HighPerformance Near-Field Reader Antennas: Comparisons of Proposed Antenna Layouts for Ultrahigh-Frequency Near-Field RadioFrequency Identification," IEEE Antennas Prop. Mag., vol. 60, no. 1, 2018.

[5] P. V. Nikitin, K. V. S. Rao, and S. Lazar, "An Overview of Near Field UHF RFID," IEEE International Conference on RFID, 2007, pp. 167174.

[6] A. Michel and P. Nepa, "UHF-RFID Desktop Reader Antennas: Performance Analysis in the Near-Field Region," IEEE Ant. Wireless Prop. Letters, vol. 15, pp. 1430 - 1433, 2016.

[7] M. Daiki, E. Perret, and S. Tedjini, "Antenna Design for UHF RFID Near-Field Applications," IEEE Int. Conf. RFID, Orlando, USA, 2013.

[8] A. L. Popov, O. G. Vendik, and N. A. Zubova, "Magnetic field intensity in near field zone of loop antenna for RFID systems," Tech. Phys. Lett., vol. 36, no. 10, pp. 882-884, Nov. 2010.

[9] X. Qing, C. K. Goh, and Z. N. Chen, “A Broadband UHF Near-Field RFID Antenna," IEEE Trans. Antennas Propag., vol. 58, no. 12, pp. 3829-3838, Dec. 2010.

[10] D. M. Dobkin, S. M. Weigand, and N. Iyer, "Segmented magnetic antennas for near-field uhf rfid.," Microw. J., vol. 50, no. 6, 2007.

[11] X. Qing, Z. N. Chen, and C. K. Goh, "UHF near-field RFID reader antenna with capacitive couplers," Electron. Lett., vol. 46, no. 24, pp. 1591-1592, 2010.

[12] P. Yang, Y. Li, L.-J. Jiang, and F. Yang, "Near-Field Loop Antenna for the UHF RFID Reader," JEST. J, vol. 9, no. 3, 2011. 
[13] Y. Zhang and J. Liu, "A new kind of low-inductance transformer type magnetic switch (TTMS) with coaxial cylindrical conductors," Rev. Sci. Instrum., vol. 84, no. 2, p. 023306, 2013.

[14] M. J. Lamm, N. Andreev, G. Ambrosio, J. Brandt, R. Coleman, D. Evbota, V. V. Kashikhin, M. Lopes, J. Miller, T. Nicol, and others, "Solenoid magnet system for the Fermilab Mu2e experiment," IEEE Trans. Appl. Supercond., vol. 22, no. 3, pp. 4100304-4100304, 2012.

[15] B. Lu and X. Yang, "Effects of High Frequency on the Distributed Parameters of the Sparse-Wound Solenoid Coils," IEEE Trans. Plasma Sci., vol. 42, no. 1, pp. 154-161, 2014.

[16] S. Lee, J. Choi, G. S. May, and I. Yun, "Modeling and analysis of 3-D solenoid embedded inductors," IEEE Trans. Electron. Packag. Manuf. , vol. 25, no. 1, pp. 34-41, 2002.

[17] C. R. Yungers, Solenoid antenna., U.S. Patent 7443362,Oct, 28, 2008

[18] D. H. Werner, "An exact integration procedure for vector potentials of thin circular loop antennas," IEEE Trans. Antennas Propag., vol. 44, no. 2, pp. 157-165, 1996.

[19] inkJet FlexThe Centre for Process Innovation. [Online]. Available: http://www.uk-cpi.com/inkjet-flex/.

[20] A. Vena, E. Perret, S. Tedjini, G. E. P. Tourtollet, A. Delattre, F. Garet, and Y. Boutant, "Design of chipless RFID tags printed on paper by flexography," IEEE Trans. Antennas Propag., vol. 61, no. 12, pp. 5868-5877, 2013.

[21] Impinj Speedway Revolution R420 UHF RFID Reader Evaluation Kit (4 Port), atlasRFIDstore. [Online]. Available: http://www.atlasrfidstore.com/impinj-speedway-revolution-r420-uhfrfid-reader-evaluation-kit-4-port/.

[22] "Blackroc Technology | Tagsys AK UHF tag (AK Tag).

[23] "Brickyard Antenna Datasheet," Impinj Support Portal. [Online]. Available: http://support.impinj.com/hc/en-us/articles/202755648Brickyard-Antenna-Datasheet. 